\title{
Differential Alternative Splicing Genes and Isoform Regulation Networks of Rapeseed (Brassica napus L.) Infected with Sclerotinia sclerotiorum
}

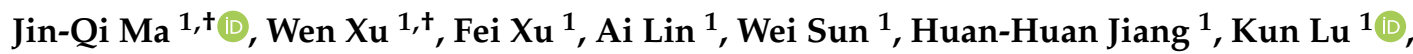 \\ Jia-Na Li ${ }^{2, *}$ and Li-Juan Wei ${ }^{1, *}$ \\ 1 College of Agronomy and Biotechnology, Southwest University, Chongqing 400715, China; \\ jinqima1996@163.com (J.-Q.M.); 18722975519@163.com (W.X.); xf9310955@163.com (F.X.); \\ ai_linnn@163.com (A.L.); reginasw@163.com (W.S.); jh13526462127@163.com (H.-H.J.); \\ drlukun@swu.edu.cn (K.L.) \\ 2 Academy of Agricultural Sciences, Southwest University, Chongqing 400715, China \\ * Correspondence: 1jn1950@swu.edu.cn (J.-N.L.); lijuanwei@swu.edu.cn (L.-J.W.); \\ Tel.: +86-023-68250642 (J.-N.L.); +86-023-68251264 (L.-J.W.); Fax: +86-023-6825070 (J.-N.L.) \\ + These authors contributed equally to this work.
}

Received: 12 June 2020; Accepted: 7 July 2020; Published: 13 July 2020

\begin{abstract}
Alternative splicing (AS) is a post-transcriptional level of gene expression regulation that increases transcriptome and proteome diversity. How the AS landscape of rapeseed (Brassica napus L.) changes in response to the fungal pathogen Sclerotinia sclerotiorum is unknown. Here, we analyzed 18 RNA-seq libraries of mock-inoculated and S. sclerotiorum-inoculated susceptible and tolerant B. napus plants. We found that infection increased AS, with intron retention being the main AS event. To determine the key genes functioning in the AS response, we performed a differential AS (DAS) analysis. We identified 79 DAS genes, including those encoding splicing factors, defense response proteins, crucial transcription factors and enzymes. We generated coexpression networks based on the splicing isoforms, rather than the genes, to explore the genes' diverse functions. Using this weighted gene coexpression network analysis alongside a gene ontology enrichment analysis, we identified 11 modules putatively involved in the pathogen defense response. Within these regulatory modules, six DAS genes (ascorbate peroxidase 1, ser/arg-rich protein 34a, unknown function 1138, nitrilase 2, v-atpase $\mathrm{f}$, and amino acid transporter 1 ) were considered to encode key isoforms involved in the defense response. This study provides insight into the post-transcriptional response of $B$. napus to $S$. sclerotiorum infection.
\end{abstract}

Keywords: Brassica napus; Sclerotinia sclerotiorum; alternative splicing; RNA-seq; WGCNA

\section{Introduction}

Rapeseed (Brassica napus L.), the second largest oilseed crop in the world [1], is vulnerable to the necrotrophic ascomycete pathogen Sclerotinia sclerotiorum, which targets over 400 host species [2,3], including soybean (Glycine max), peanut (Arachis hypogaea), and sunflower (Helianthus annuus) [4]. S. sclerotiorum infects the leaves and stem of its host plants and causes Sclerotinia stem rot (SSR), also known as white mold, in rapeseed, resulting in yield losses of up to $94 \%$ in severe SSR outbreak seasons in the major growing areas such as Canada, China and the USA [5,6]. Despite this huge economic impact, no highly resistant $B$. napus varieties have been developed.

Current research into SSR is mainly focused on the pathogenicity and resistance mechanisms involved. The outcome of SSR is the product of the interaction of multiple factors in both B. napus and S. sclerotiorum, resulting in a gradient of resistance phenotypes termed quantitative disease resistance [7-9]. The resistance 
strategy has been frequently explored using approaches such as genome-wide association studies [10], quantitative trait locus mapping [11], RNA sequencing (RNA-seq) [12] and the identification of relevant noncoding RNAs [13], resulting in the discovery of rare but highly effective factors. Previous RNA-seq studies mainly focused on e transcriptional response and differential expression analyses. However, here, we use RNA-seq data to analyze alternative splicing (AS) to shed light on B. napus resistance to SSR.

AS is a type of post-transcriptional regulation that produces various mRNA transcripts from precursor mRNAs (pre-mRNAs) of a single gene, increasing transcriptomic and proteomic diversity [14]. AS occurs in more than $95 \%$ of intron-containing genes in humans [15] and ca. 33-60\% of these genes in plants $[16,17]$, with the types of AS including exon skipping (ES), intron retention (IR), alternative $5^{\prime}$ splice sites, alternative $3^{\prime}$ splice sites, mutually exclusive $3^{\prime}$ untranslated regions, tandem untranslated regions, mutually exclusive $5^{\prime}$ exons and mutually exclusive exons [18]. Although the ratios of these different AS types vary among different species, plant AS events predominantly involve IR, which is the least common type in animals [19].

AS occurs following the recognition by the spliceosome, containing small nuclear RNAs, of four cis-acting sequences: the exon-intron junctions at the $5^{\prime}$ and $3^{\prime}$ ends of the introns, the BRANCH-SITE sequence, and the polypyrimidine tract [20]. Strong recognition of certain splice sites usually results in constitutive splicing [21], which is generally based on the GU-AG rule [22]. The selection of splicing sites leading to AS is determined by RNA-binding proteins [23], mainly serine/arginine-rich (SR) proteins and heterogeneous nuclear ribonucleoprotein particle proteins, which recruit the spliceosome to bind to the splicing regulatory elements, such as the stimulating (exonic/intronic splicing enhancers) or inhibiting (exonic/intronic splicing silencers) splice sites [24].

AS affects the biology of a cell or organism by causing changes in mRNA stability, mRNA localization and mRNA translation, which further leads to noncoding transcripts or differently functional proteins and other biochemical properties [25]. AS may be associated with 15\% of genetic diseases in humans [26]. However, it can also play important roles in plant development and stress response. In the area of pathogen defense, the AS of many $R$ genes, including TIR-NBS-LRR, Arabidopsis RPS4, Medicago truncatula RCT1, Flax L6, Tomato Bs4, CC-NBS-LRR and so on, is required for the defense response [27] such as the production of the shorter and longer transcripts of the tobacco (Nicotiana sp.) $N$ gene for resistance to the tobacco mosaic virus [28]. The diverse AS isoforms of Arabidopsis thaliana resistance to pseudomonas syringae 4 provide resistance to tomato strain DC3000 [29], whereas the diverse AS isoforms of OsWRKY62 and OsWRKY76 in rice (Oryza sativa) convey resistance against the pathogens Magnaporthe oryzae and Xanthomonas oryzae pv. oryzae [30]. In addition to identifying single AS events in a few genes in the past, recent studies have analyzed massive AS events and explored genome-wide AS networks; for example, massive and rapid AS responses were identified in the first few hours of cold stress in A. thaliana [14], while in the model grass Brachypodium distachyon, infection by the panicum mosaic virus and its satellite virus significantly increased the number of AS events [19]. The AS landscape in B. napus in response to $S$. sclerotiorum has not been reported.

In this study, we performed a differential AS analysis of B. napus RNA-seq data to explore candidate resistant genes in response to a $S$. sclerotiorum infection. We used coexpression analysis to identify the regulatory networks of the AS isoforms based on isoform expression levels rather than gene expression levels. This study elucidated the dynamic response of AS isoforms to enhance our understanding of the B. napus response to S. sclerotiorum.

\section{Materials and Methods}

\subsection{Transcriptome Analysis}

The RNA-seq data of B. napus before and after inoculation with S. sclerotiorum were downloaded from the GEO database (https://www.ncbi.nlm.nih.gov/geo/; accession numbers SRR3537150, SRR3537151，SRR3537153，SRR3537154，SRR3537155，SRR3537156， SRR3537159， SRR3537160, 
SRR1793826， SRR1793858，SRR1793863，SRR1793864，SRR1793865，SRR1793876， SRR1793909， SRR1793924, SRR1793925, and SRR1793927) [12,31]. The raw data were converted to the FASTQ format using the SRA Toolkit version 2.9.0 (https://www.ncbi.nlm.nih.gov/books/NBK158900/), and quality trimming and adapter clipping were then performed using Trimmomatic version 0.36 [32] (modified parameters: ILLUMINACLIP = TruSeq3-PE.fa:2:30:10 or ILLUMINACLIP = TruSeq3-SE.fa:2:30:10, LEADING $=3$, TRAILING $=3$, SLIDINGWINDOW $=4: 15$, and HEADCROP $=10$ ). By generating the index using the chromosome sequence from B. napus Genome Browser (http://www.genoscope.cns.fr/brassicanapus/) [1], the prefiltered reads were mapped to the B. napus reference genome using STAR version 2.5.3a (with sjdbOverhang $=150$ ) [33]. The mapped reads were assembled into putative transcripts using Cufflinks version 2.2.1 [34], then were tracked across all samples using Cuffcompare to estimate their relative abundances.

\subsection{AS Landscape and Differential AS (DAS) Analysis}

Using the gtf annotation obtained from Cufflinks, the AS landscape (intron retention, alternate acceptors, alternate donors, exon skipping, and other splicing events) of every sample was analyzed using Astalavista version 4.0 [35]. The DAS genes between different cultivars and different treatments were respectively quantified using rMATS (with cstat $=0.0001$, tstat $=6$, FDR $<0.01$ ) [36], and were then visualized using rmats2sashimiplot-master (https://github.com/Xinglab/rmats2sashimiplot).

\subsection{Weighted Gene CoExpression Network Analysis (WGCNA) of Isoforms}

To further explore the regulatory relationship of each isoform, a WGCNA $[37,38]$ run in R version 3.4.4 was used to perform data cleaning (the top $14 \%$ transcripts were selected based on their fragments per kilobase of transcript per million mapped reads (FPKM) variance analysis), sample clustering, soft threshold filtering (power $=\mathrm{sft} \$$ powerEstimate), scale-free check (scaleFreePlot), one-step network construction (TOMType $=$ "unsigned", mergeCutHeight $=0.25$, verbose $=3$ ), module identification, and network visualizing (random selection of 1000 transcripts). The weighted values of each relationship between two coexpressed transcripts gained from each module were filtered with $>0.15$ to obtain the significantly coexpressed networks using Cytoscape version 3.5.1 [39]. Using R, the isoform heatmap was constructed on the average FPKM values of the biological replicates, which were transformed into $\log _{2}(\mathrm{FPKM}+1)$ values.

\subsection{Functional Enrichment and Clustering}

To determine the potential function of each module, a gene ontology (GO) enrichment analysis was performed using the R package topGO [40]. The modules containing fungus relative terms with classicFisher $<0.01$ were selected as target modules, such as response to fungus and defense to fungus. A GO enrichment Senior Bubble plot was drawn by the OmicShare tools (http://www.omicshare.com/ tools).

\subsection{Homologous Gene Identification}

Based on the studies showing that $A$. thaliana and B. napus have a common ancestor in the Brassicaceae [41,42], the arabidopsis genes homologous to the BnDAS genes were identified using BLASTP with an $E$ value of $\leq \mathrm{e}^{-5}$ using the NCBI BLAST version 2.2.30+ program [43].

\section{Results}

\subsection{AS Landscape in B. napus in Response to S. sclerotiorum}

To identify the AS landscape of B. napus in response to a S. sclerotiorum infection, a total of 18 RNA-seq libraries were analyzed in this study (Table 1). The raw data were downloaded from two datasets, one of which comprised single-end reads and the other paired-end reads. The first dataset contained eight RNA-seq libraries from the leaves of two B. napus cultivars (susceptible Westar and 
resistant ZY821) at $24 \mathrm{~h}$ postinoculation (hpi) with S. sclerotiorum or a mock treatment. The second dataset contained ten libraries from the stems of two B. napus cultivars (susceptible J902 and resistant J964) subjected to a mock treatment or at 24 and $48 \mathrm{hpi}$ with $S$. sclerotiorum. Based on the reference genome [1], we performed a quality trim, genome mapping, transcript assembly and transcript merging to identify the FPKM of every isoform (Table S1). For the single-end transcriptome data, $70.31 \%$ of the reads were uniquely mapped to the reference genome and the average mapped read length was $96.32 \mathrm{bp}$. For the paired-end transcriptome data, $82.55 \%$ of the reads were uniquely mapped to the reference genome and the average mapped read length was $178.26 \mathrm{bp}$ (Table S2).

The assembled transcript annotation file was further used to identify the AS landscape using Astalavista. As shown in Figure 1 and Table S3, the results indicated that IR was the most common AS event compared with the AA (alternate acceptors), AD (alternate donor), and ES. In addition, we computed the AS event frequency of the merged transcripts (overall AS events/merged transcript number). Compared with the mock treatment (Figure 1a,c,e,g), the AS ratio was slightly increased in the 24 hpi plants (Figure 1b,d,f,h), whereas the AS ratio was decreased in the 48 hpi treatment (Figure 1i). This indicated that S. sclerotiorum triggered AS at the early stages of infection.
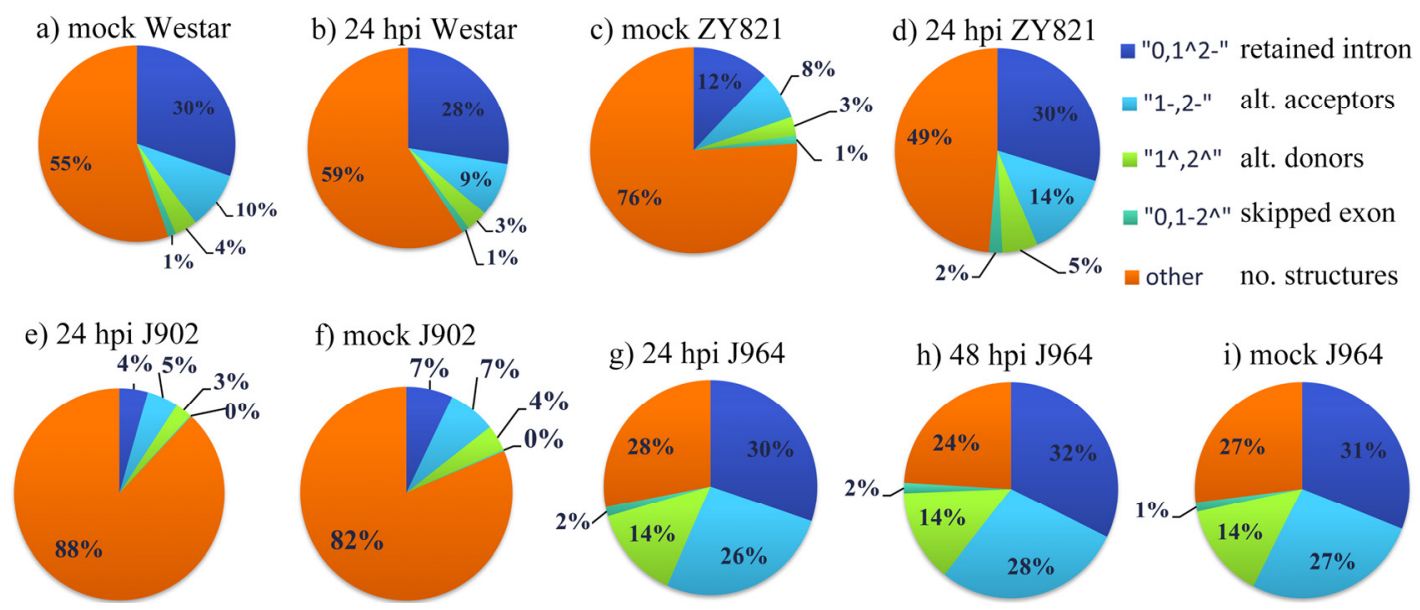

Figure 1. AS Landscapes of healthy and pathogen-inoculated B. napus. Frequency of AS types of mock $(\mathbf{a}, \mathbf{c})$ and $24 \mathrm{hpi}(\mathbf{b}, \mathbf{d})$ in B. napus susceptible (cv. Westar) and tolerant (cv. ZY821) genotypes. Frequency of AS types of mock (f), 24 hpi (e) in B. napus susceptible (cv. J902) and mock (i), 24 hpi (g), 48 hpi (h) in B. napus tolerant (cv. J964) genotypes.

\subsection{Identification of DAS Genes}

In total, 160 DAS genes were identified from the paired-end RNA libraries using rMATS, whereas no DAS genes were identified from the single-end data. We filtered the 160 DAS genes using a false discovery rate $(F D R)<0.05$, resulting in the identification of 79 important DAS genes. Using a differential analysis of the mock-treated and infected materials, the number of infection-induced DAS genes in the comparisons J2_24 h vs. J2_ck, J4_24 h vs. J4_ck, and J4_48 h vs. J4_ck were, respectively, 22,6 , and 10 (Table S4). Differential analysis between the resistant and susceptible cultivars (J2_24 h vs. J4_24 h) after inoculation with $S$. sclerotiorum led to the identification of 49 cultivar-related DAS genes. Eight overlapping DAS genes were detected in both infection-induced and cultivar-related DAS genes.

Homologs of 68 of the B. napus DAS genes were identified in arabidopsis (Table 3), which enabled their annotation; the functions of the other 11 B. napus DAS genes remained unknown. In addition, 27 of the arabidopsis homologous genes were previously shown to be AS genes, according to the ASIP database [44]. Based on the GO enrichment analysis, the 79 significantly DAS genes were involved in a variety of functions including the valine catabolic process, nuclear retention of unspliced pre-mRNA, nuclear mRNA surveillance of the spliceosome and fatty acid $\beta$-oxidation (Figure S1 and Table S5). 
Table 1. Statistical summary of RNA-seq material and the detected alternative splicing (AS) pattern.

\begin{tabular}{|c|c|c|c|c|c|c|c|c|c|c|c|c|}
\hline \multirow{2}{*}{ Cultivar } & \multirow{2}{*}{ Treatment } & \multirow{2}{*}{$\begin{array}{l}\text { Dataset } \\
\text { Name }\end{array}$} & \multirow{2}{*}{$\begin{array}{l}\text { Data } \\
\text { Type }\end{array}$} & \multirow{2}{*}{ Number } & \multirow{2}{*}{$\begin{array}{l}\text { Overall AS } \\
\text { Events }\end{array}$} & \multirow{2}{*}{$\begin{array}{c}\text { Merged } \\
\text { Transcript-Number }\end{array}$} & \multirow{2}{*}{$\begin{array}{c}\text { AS Event } \\
\text { Ratio }\end{array}$} & \multicolumn{5}{|c|}{ AS Type } \\
\hline & & & & & & & & IR & AA & AD & ES & Other Event \\
\hline Westar & Mock & W_ck & SE & SRR3537150, SRR3537151 & 28,518 & 585,673 & $4.87 \%$ & 8638 & 2724 & 1049 & 380 & 15,727 \\
\hline Westar & $24 \mathrm{hpi}$ & W_24h $\mathrm{h}$ & SE & SRR3537153, SRR3537154, SRR3537155 & 44,388 & 784,570 & $5.66 \%$ & 12,230 & 3860 & 1538 & 518 & 26,242 \\
\hline ZY 821 & Mock & Z_ck & SE & SRR3537156 & 7997 & 234,401 & $3.41 \%$ & 964 & 617 & 237 & 100 & 6079 \\
\hline ZY 821 & 24 hpi & $\mathrm{Z}_{-} 24 \mathrm{~h}$ & SE & SRR3537159, SRR3537160 & 16,770 & 467,414 & $3.59 \%$ & 4993 & 2326 & 934 & 358 & 8159 \\
\hline $\mathrm{J} 902$ & Mock & J2_ck & PE & SRR1793858 & 34,734 & 388,008 & $8.95 \%$ & 2470 & 2538 & 1371 & 79 & 28,276 \\
\hline $\mathrm{J} 902$ & 24 hpi & $\mathrm{J} 2 \_24 \mathrm{~h}$ & PE & SRR1793826 & 69,013 & 536,471 & $12.86 \%$ & 3038 & 3311 & 1792 & 130 & 60,742 \\
\hline J964 & Mock & J4_ck & PE & SRR1793924, SRR1793925, SRR1793927 & 32,750 & 622,164 & $5.26 \%$ & 10,156 & 8688 & 4606 & 453 & 8847 \\
\hline J964 & $24 \mathrm{hpi}$ & J4_24 h & PE & SRR1793863, SRR1793864, SRR1793865 & 34,397 & 639,525 & $5.38 \%$ & 10,405 & 9084 & 4759 & 489 & 9660 \\
\hline J964 & 48 hpi & J4_ $48 \mathrm{~h}$ & $\mathrm{PE}$ & SRR1793876, SRR1793909 & 15,202 & 387,918 & $3.92 \%$ & 4912 & 4308 & 2087 & 239 & 3656 \\
\hline
\end{tabular}


Table 2. Annotation of the 79 DAS genes in B. napus.

\begin{tabular}{|c|c|c|c|c|c|}
\hline DAS Comparison & DAS Gene & At Ortholog & ASIP Synonym & \% Identity & At Ortholog Family \\
\hline \multirow{18}{*}{ J2_24 h vs. J2_ck } & BnaC05g00740D & AT1G01170 & T25K16.16 & 92.50 & Protein of unknown function (DUF1138) \\
\hline & BnaA05g20710D & AT1G33970 & F12G12.21 & 82.22 & Immune-associated nucleotide binding 9 \\
\hline & BnaA07g03760D & AT1G64610 & & 57.47 & Transducin/WD40 repeat-like superfamily protein \\
\hline & BnaA07g25520D & AT1G65950 & F12P19.11 & 87.07 & Protein kinase superfamily protein \\
\hline & BnaA09g18680D & AT2G01540 & & 94.67 & Calcium-dependent lipid-binding protein \\
\hline & BnaA02g26350D & AT2G02360 & & 61.11 & Phloem protein 2-B10 \\
\hline & BnaA07g13990D & AT2G28550 & T17D12.11 & 80.88 & (TF) Related to AP2.7 \\
\hline & BnaC04g04760D & AT2G46090 & T3F17.26 & 84.18 & Diacylglycerol kinase protein \\
\hline & BnaC03g25720D & AT2G46340 & & 77.77 & SPA (suppressor of phyA-105) protein \\
\hline & BnaC01g36600D & AT3G14770 & T21E2.6 & 83.47 & Vacuolar sugar transporter SWEET2 \\
\hline & BnaCnng45490D & AT4G16845 & FCAALL.23 & 80.65 & VEFS-Box of polycomb protein \\
\hline & BnaCnn1978 & AT4G35785 & F4B14.7 & 68.05 & RNA-binding (RRM/RBD/RNP motifs) protein \\
\hline & BnaA03g06980D & AT5G18230 & & 88.12 & Transcription regulator NOT2/NOT3/NOT5 protein \\
\hline & BnaA10g14420D & AT5G21170 & T10F18.200 & 80.12 & $5^{\prime}$-AMP-activated protein kinase $\beta-2$ subunit protein \\
\hline & BnaA03g09910D & AT5G58110 & & 88.38 & ATPase activators \\
\hline & BnaA06g22260D & AT5G62760 & & 71.72 & P-loop containing nucleoside triphosphate hydrolases superfamily \\
\hline & BnaC09g07670D & AT5G66890 & & 73.10 & Leucine-rich repeat (LRR) protein \\
\hline & BnaC09g23900D & unknown & & & \\
\hline \multirow{4}{*}{$\begin{array}{l}\text { J2_24 h vs. J2_ck } \\
\text { J2_24 h vs. J4_24 h }\end{array}$} & BnaA09g50970D & AT1G03140 & & 79.02 & Splicing factor Prp18 protein \\
\hline & BnaC09g09280D & AT1G75660 & F10A5.15 & 68.57 & $5^{\prime}-3^{\prime}$ exoribonuclease 3 \\
\hline & BnaA06g38980D & AT3G44300 & & 81.42 & Nitrilase 2 \\
\hline & BnaC04g40450D & AT5G65940 & K14B20.11 & 80.00 & $\beta$-hydroxyisobutyryl-CoA hydrolase 1 \\
\hline \multirow{17}{*}{ J2_24 h vs. J4_24 h } & BnaCnng45130D & AT1G02305 & & 78.82 & Cysteine proteinases superfamily protein \\
\hline & BnaC05g05550D & AT1G07890 & F24B9.2 & 92.00 & Ascorbate peroxidase 1 \\
\hline & BnaC05g22390D & AT1G29350 & & 74.24 & RNA polymerase II degradation factor-like protein (DUF1296) \\
\hline & BnaA06n0344 & AT1G48840 & & 50.79 & Plant protein of unknown function (DUF639) \\
\hline & BnaC06g04140D & AT1G50910 & & 79.28 & Hypothetical protein \\
\hline & BnaA02g12910D & AT1G67170 & & 69.08 & Sarcolemmal membrane-associated protein \\
\hline & BnaA07g24840D & AT1G68010 & T23K23.14 & 96.90 & Hydroxypyruvate reductase \\
\hline & BnaCnng00710D & AT2G04560 & & 82.68 & Transferases \\
\hline & BnaCnng63360D & AT2G19480 & F3P11.8 & 84.7 & Nucleosome assembly protein $1 ; 2$ \\
\hline & BnaC04g33610D & AT2G21270 & & 80.73 & Ubiquitin fusion degradation 1 \\
\hline & BnaAnng26570D & AT2G23010 & & 77.02 & Serine carboxypeptidase-like 9 \\
\hline & BnaC04g45930D & AT2G26900 & & 89.86 & Sodium Bile acid symporter family \\
\hline & BnaC04g47700D & AT2G41460 & & 69.98 & Apurinic endonuclease-redox protein \\
\hline & BnaC04g03300D & AT2G44065 & & 90.23 & Ribosomal protein L2 family \\
\hline & BnaA03g30650D & AT3G09500 & & 94.31 & Ribosomal L29 protein \\
\hline & BnaAnng09240D & AT3G12130 & & 78.88 & KH domain-containing protein/zinc finger (CCCH type) family \\
\hline & BnaC09g01340D & AT3G27100 & MOJ10.18 & 87.50 & ENY2 \\
\hline
\end{tabular}


Table 3. Annotation of the 79 DAS genes in B. napus.

\begin{tabular}{|c|c|c|c|c|c|}
\hline DAS Comparison & DAS Gene & At Ortholog & ASIP Synonym & $\%$ Identity & At Ortholog Family \\
\hline \multirow{24}{*}{ J2_24 h vs. J4_24 h } & BnaC02g09410D & AT3G44280 & \multirow{5}{*}{ T14E10.50 } & 73.15 & \multirow{2}{*}{$\begin{array}{l}\text { Peptidyl-prolyl cis-trans isomerase G } \\
\alpha \text {-glucan phosphorylase } 2\end{array}$} \\
\hline & BnaA06g17710D & AT3G46970 & & 95.01 & \\
\hline & BnaA04g04080D & AT3G54480 & & 85.87 & SKP1/ASK-interacting protein 5 \\
\hline & BnaC09g00230D & AT4G02780 & & 85.71 & Terpenoid cyclases/Protein prenyltransferases superfamily \\
\hline & BnaA05n0101 & AT4G15090 & & 84.82 & (TF) FRS (FAR1 Related Sequences) family \\
\hline & BnaA01g17760D & AT4G17300 & \multirow{3}{*}{$\begin{array}{l}\text { MHF15.4 } \\
\text { MLE8.1 }\end{array}$} & 90.49 & Class II aminoacyl-tRNA and biotin synthetases superfamily \\
\hline & BnaC03g02590D & AT5G06440 & & 61.86 & Polyketide cyclase/dehydrase/lipid transport superfamily protein \\
\hline & BnaA06g26720D & AT5G24150 & & 77.76 & FAD/NAD(P)-binding oxidoreductase protein \\
\hline & BnaA07g15350D & AT5G41350 & & 79.44 & \\
\hline & BnaA02g22910D & AT5G42210 & \multirow{3}{*}{ MQL5.3 } & 81.51 & Major facilitator superfamily protein \\
\hline & BnaA02g25070D & AT5G47180 & & 94.09 & Plant VAMP (vesicle-associated membrane protein) family \\
\hline & BnaC09g28380D & AT5G52380 & & 69.28 & \multirow{2}{*}{$\begin{array}{l}\text { Vascular-related nac-domain } 6 \\
\beta \text {-amylase } 4\end{array}$} \\
\hline & BnaC03g14120D & AT5G55700 & & 89.87 & \\
\hline & BnaC09g33270D & AT5G56740 & \multirow{11}{*}{ K18B18.4 } & 77.87 & Histone acetyltransferase of the GNAT family 2 \\
\hline & BnaC02g10170D & AT5G59050 & & 77.24 & $\mathrm{G}$ patch domain protein \\
\hline & BnaC07g16670D & \multirow{2}{*}{$\begin{array}{l}\text { AT5G66550 } \\
\text { unknown }\end{array}$} & & \multirow{9}{*}{80.10} & \multirow{9}{*}{ Maf-like protein } \\
\hline & BnaCnng08340D & & & & \\
\hline & BnaA06g40000D & unknown & & & \\
\hline & BnaC07n0282 & unknown & & & \\
\hline & BnaCnn1013 & unknown & & & \\
\hline & BnaC02g22380D & unknown & & & \\
\hline & BnaA06n0074 & unknown & & & \\
\hline & BnaC07n0181 & unknown & & & \\
\hline & BnaA03n0594 & unknown & & & \\
\hline \multirow{2}{*}{$\begin{array}{l}\text { J2_24 h vs. J4_24 } \\
\text { h \J4_24 h vs. J4_ck }\end{array}$} & BnaA09 g19610D & AT3G25840 & \multirow{2}{*}{ K9I22.6 } & 73.57 & \multirow{2}{*}{$\begin{array}{l}\text { Protein kinase superfamily protein } \\
\text { Amino acid transporter } 1\end{array}$} \\
\hline & BnaA03g58530D & AT4G21120 & & 90.05 & \\
\hline $\begin{array}{l}\text { J2_24 h vs. J4_24 } \\
\text { h } \backslash J 4 \_48 \text { h vs. J4_ck }\end{array}$ & BnaC02g27060D & AT4G02620 & & 87.23 & Vacuolar ATPase subunit F protein \\
\hline \multirow{4}{*}{ J4_24 h vs. J4_ck } & BnaC06g34460D & AT1G73650 & \multirow[t]{2}{*}{ F25P22.29 } & 88.66 & Protein of unknown function (DUF1295) \\
\hline & BnaCnng43580D & AT3G21865 & & 82.39 & Peroxin 22 \\
\hline & BnaCnng19550D & AT5G57860 & MTI20.11 & 87.37 & Ubiquitin-like superfamily protein \\
\hline & BnaA04g17110D & AT5G65940 & K14B20.11 & 80.52 & $\beta$-hydroxyisobutyryl-CoA hydrolase 1 \\
\hline \multirow{9}{*}{ J4_48 h vs. J4_ck } & BnaC08g42380D & AT1G10130 & \multirow[t]{2}{*}{ T27I1.16 } & 95.79 & \multirow{2}{*}{$\begin{array}{l}\text { Endoplasmic reticulum-type calcium-transporting ATPase } 3 \\
\text { F-box/associated interaction domain protein }\end{array}$} \\
\hline & BnaC03g69240D & AT1G52490 & & 47.73 & \\
\hline & BnaC09n0143 & AT1G62800 & \multirow[t]{2}{*}{ F23N19.17 } & 80.31 & \multirow{2}{*}{$\begin{array}{l}\text { Aspartate aminotransferase } 4 \\
\text { Zinc knuckle (CCHC-type) protein }\end{array}$} \\
\hline & BnaAnng09100D & AT1G75560 & & 85.41 & \\
\hline & BnaC08g21130D & AT3G49430 & \multirow[t]{5}{*}{ T9C5.30 } & 85.21 & Ser/Arg-rich protein $34 \mathrm{~A}$ \\
\hline & BnaC07g01360D & AT5G03495 & & 30.87 & \multirow{4}{*}{$\begin{array}{l}\text { RNA-binding (RRM/RBD/RNP motifs) protein } \\
\text { Immunoglobulin E-set superfamily protein }\end{array}$} \\
\hline & BnaC04g37090D & AT5G06480 & & 69.11 & \\
\hline & BnaC08g02660D & unknown & & & \\
\hline & BnaC09n0005 & unknown & & & \\
\hline
\end{tabular}


Based on the annotated functions of the homologous genes in A. thaliana, four of the 79 DAS genes were putatively related to RNA splicing, including BnaCnn1978 (RNA-binding protein), BnaC08g21130D (SR protein 34a), and BnaC07g01360D (RNA-binding protein). One gene (BnaA09g50970D, splicing factor PRP18 protein), which was previously shown to be involved in promoting splicing at weak splice sites [45], was identified in both the infection-induced and cultivar-related DAS gene groups. Three of the 79 DAS genes were known to be related to the defense response, including BnaA05g20710D (immune-associated nucleotide binding 9), which encodes a plasma membrane-localized protein that functions as a negative regulator of basal immunity in A. thaliana [46]; BnaC09g07670D (LRR [Leucine-rich repeat] protein), which is reported likely as a pseudogene with its two paralogs transducing the defense signal downstream of TNL(TIR -type sensor NLR) in A. thaliana [47]; and BnaC04g37090D (immunoglobulin E-set superfamily protein). Two genes, BnaA07g13990D (encoding a protein related to AP2.7) and BnaA03g06980D (transcription regulator NOT2/NOT3/NOT5 protein), were believed to be involved in transcriptional regulation. The cultivar-related AS genes were BnaC05g22390D (RNA polymerase II degradation factor-like protein) and BnaA05n0101 (FRS [FAR1-RELATED SEQUENCE] family protein). In addition, we identified DAS genes encoding some important enzymes, such as protein kinases, nitrilase and transferases.

\subsection{WGCNA of All Isoforms}

WGCNA is used to describe gene correlation patterns and identify key genes by detecting gene modules, intramodular connectivity, gene significance and other features [38]. To explore the potential function of the DAS genes and analyze the diverse functions of the different isoforms encoded by a single gene, we performed a WGCNA using 575,171 isoforms (Figure 2). The FPKM values of all transcripts, including all isoforms, were next used to generate the coexpression network.

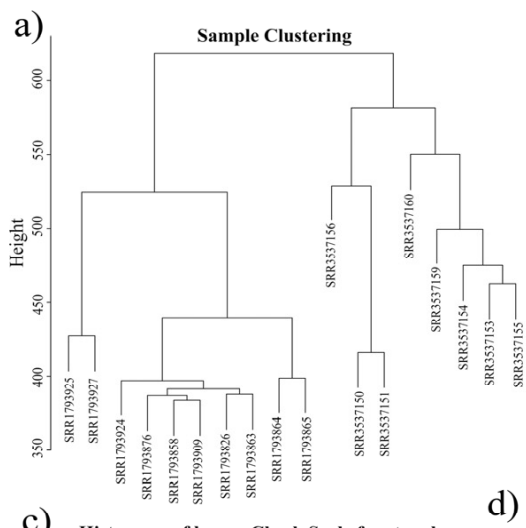

c) Histogram of $k$ Check Scale free topology

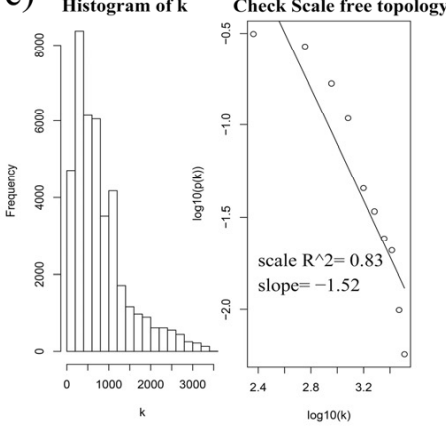

b)

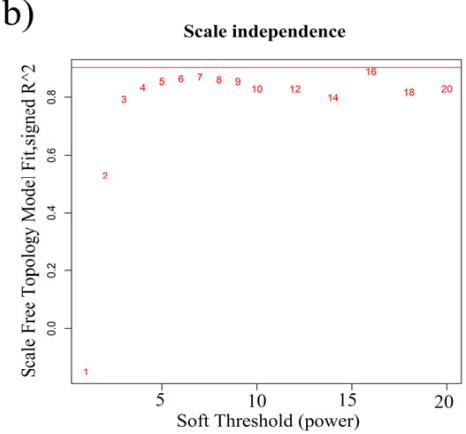

d)

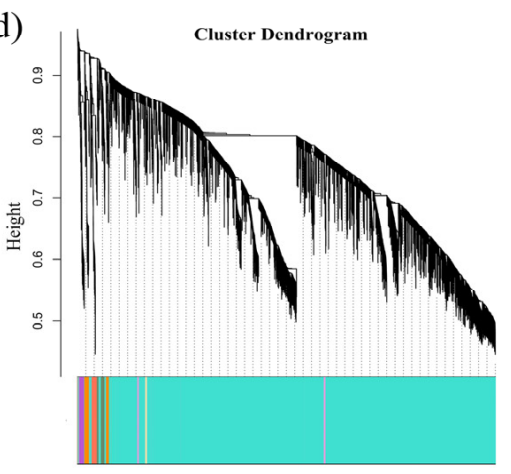

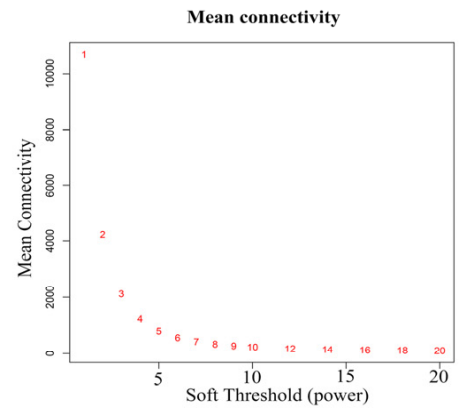

e)

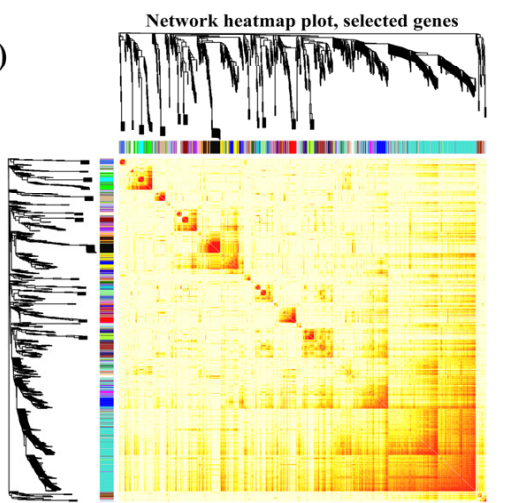

Figure 2. Coexpression network generation in isoform level by WGCNA. (a) Sample clustering with the FPKM of all isoforms from eighteen RNA-seq libraries. (b) Soft threshold selection based on the recommended power. (c) Scale free topology check with coefficient of determination $\left(R^{2}\right)$ equal to 0.83. (d) Cluster dendrogram of all isoforms by dissimilarity to obtain 85 modules. Each vertical line represents a single isoform. (e) Network heatmap of 1000 randomly selected isoforms. 
We performed a variance analysis to remain 40,441 isoforms so that the soft threshold could be up to five. Based on the distribution that approximately followed a straight line (Figure 2c), the network was a scale-free topology, meaning it was suitable for use in the coexpression analysis. We identified 85 modules (Table S6), then randomly selected 1000 isoforms to plot the network heatmap.

We performed a GO analysis on each of the identified modules (Figure S2 and Table S7), and 11 modules were selected for a further analysis to identify the response-related isoforms based on the GO terms and defense response (Figure 3). Among the 11 pathogen-related modules, we identified 25 response-related isoforms whose precursor genes were identified as 19 DAS genes (Figure 4, Table S8). Three of these 19 genes (BnaC05g05550D, BnaC08g21130D, and BnaC05g00740D) encoded nine isoforms that were identified in several modules, which suggested that these genes might play crucial roles in different response-related networks by producing different isoforms. In addition, another three overlapping DAS genes (BnaA06g38980D, BnaC02g27060D, and BnaA03g58530D) were identified as being differentially spliced in both infection- and cultivar-specific manners. These six DAS genes were considered to be the key candidate defense genes on which to perform further analysis.
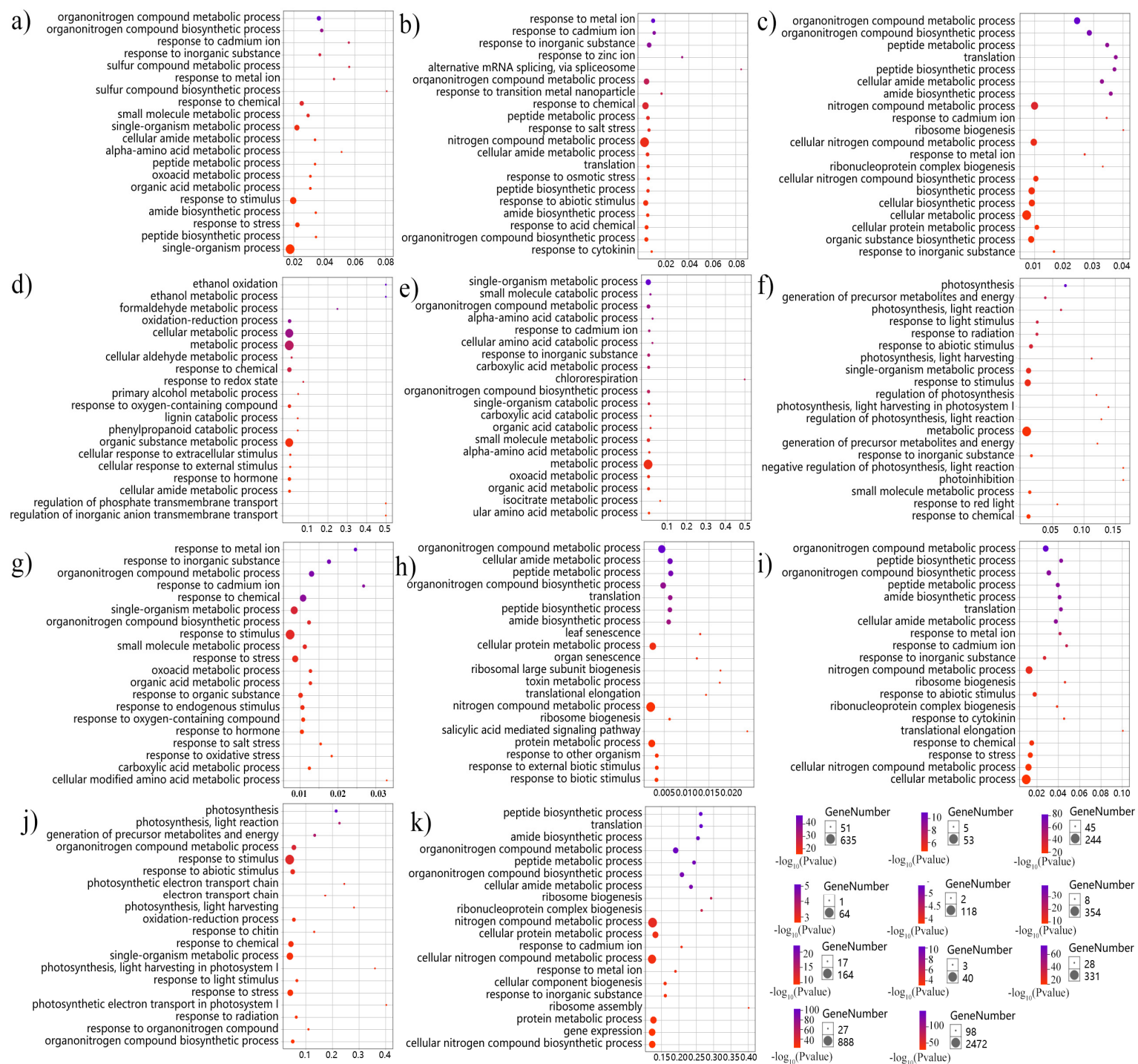

Figure 3. Top 20 of GO terms enrichment Senior Bubble of the eleven defense-related modules. (a-k) represents black, plum2, royalblue, coral1, yellowgreen, tan, lightyellow, darkseagreen4, cyan, blue, turquoise module. The $x$-axis represents the RichFactor which shows the enrichment level. The size of the bubble represents the enriched gene number. 
a)

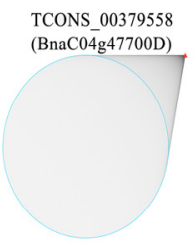

e)

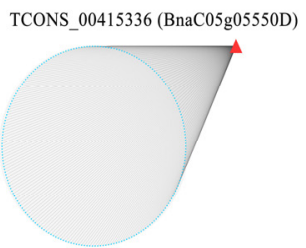

i)

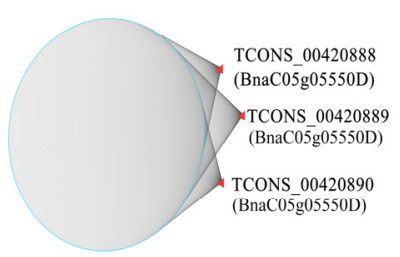

b)

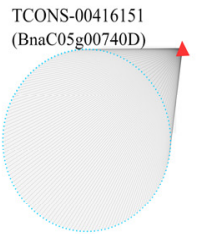

f)

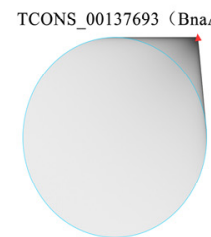

j)

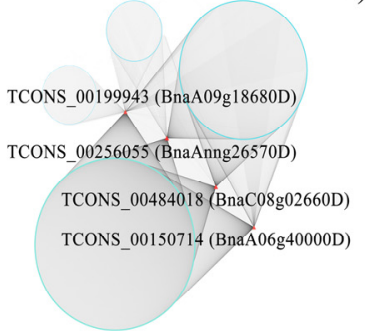

c)

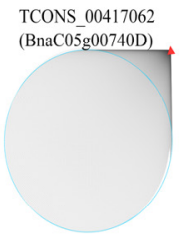

g)

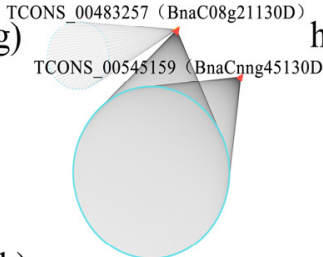

k)

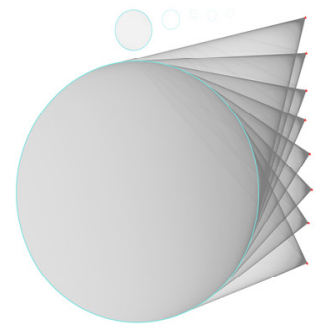

d)

TCONS_0016649 (BuaA07g24840D)

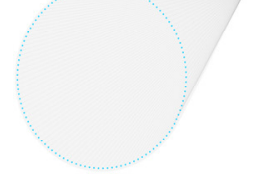

h)

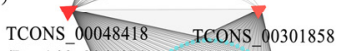
$(\mathrm{BnaA03} \mathrm{g} 30650 \mathrm{D}) \quad(\mathrm{BnaC} 02 \mathrm{~g} 27060 \mathrm{D})$

Figure 4. Coexpression networks of DAS genes in eleven defense-related modules. (a-k) represents black, plum2, royalblue, coral1, yellowgreen, tan, lightyellow, darkseagreen4, cyan, blue, turquoise module. Using Cytoscape, the red triangles represent the involved isoforms of the DAS genes, with the blue nodes representing connected isoforms.

\subsection{The Isoform Expression Landscape of Crucial DAS Genes}

To further elucidate the function of the above six candidate genes, we illustrated their DAS pattern in terms of their genomic coordinates and the expression pattern of their isoforms. In Figure 5, the reads of the ES isoforms and exon inclusion isoforms were quantified for each candidate gene to determine inclusion levels (IncLevels), representing the normalized proportion of exon inclusion events. In addition to illustrating the proportion of AS events, Figure 5 also provides modified values for the number of reads per kilobase of transcript per million mapped reads (RPKM) (y axis) of every site along the genomic coordinate ( $x$ axis) and the junction reads (the number labeled on the line) to present the read density [48].

BnaC05g05550D is homologous to AT1G07890, which encodes ascorbate peroxidase 1 (APX1). This gene was DAS in the susceptible and resistant cultivars at 24 hpi with S. sclerotiorum (Figure 5a). In susceptible cultivar J2, the IncLevel of BnaC05g05550D was much lower than it was in the resistant cultivar J4, whereas the first and the third exons were expressed to a much greater level. This suggests that, in the resistant cultivar, APX1 tended to reduce the ES events so that as many exons as possible were retained in the transcripts, whereas the susceptible cultivar tended to skip its second exon. Expression heatmaps of all 13 isoforms of APX1 were made (Figure 6a), which were differently abundant in the different conditions analyzed. Only five isoforms (TCONS_00410992, TCONS_00415336, TCONS_00420888, TCONS_00420889, and TCONS_00420890) were involved in the defense-related modules (turquoise, yellow-green, and cyan modules). This suggested that these five defense-related isoforms may function differently in the two cultivars when infected with S. sclerotiorum, resulting in differences in their resistance levels to this pathogen.

BnaC08g21130D is homologous to AT3G49430, which encodes SR34a. This gene was DAS in the mock-treated and infected resistant cultivar plants at $48 \mathrm{hpi}$ (Figure $5 \mathrm{~b}$ ). In the infected material, the IncLevel was much lower than in the mock-treated material, and the first and third exons were expressed to a much higher level. This suggests that, in response to the infection, the ES events in 
$S R 34 a$ were increased, with the second exon being skipped. Expression heatmaps were made of all 19 isoforms of $S R 34$ a (Figure 6b). Only five isoforms were identified in the two different materials above, with the TCONS_00494944 isoform involved in a defense-related network (turquoise). This suggested that the five isoforms might respond to the infection in the resistant cultivar after $48 \mathrm{~h}$.

BnaC05g00740D is homologous to AT1G01170, which encodes the protein of unknown function 1138. The BnaC05g00740D transcripts were differentially alternatively spliced in the mock-treated and infected susceptible cultivar at $24 \mathrm{hpi}$ (Figure 5c). Under the infection, the IncLevel was much higher than in the mock-treated plants, suggesting that BnaC05g00740D reduces the ES events to retain all the exons at $24 \mathrm{hpi}$. Expression heatmaps were made of all eight isoforms of BnaC05g00740D (Figure 6c). Four of the isoforms showed different abundances in the different experimental conditions. The isoforms associated with the defense-related network were not significantly differently abundant between the different conditions, however. This suggested that the four differently abundant isoforms may have novel roles in responding to $S$. sclerotiorum infection.

a)
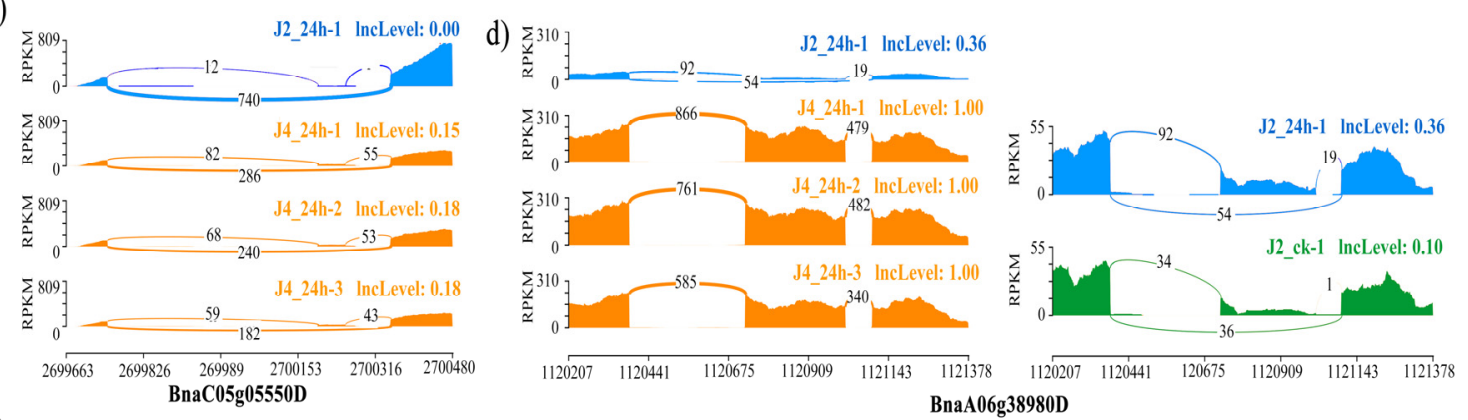

b)

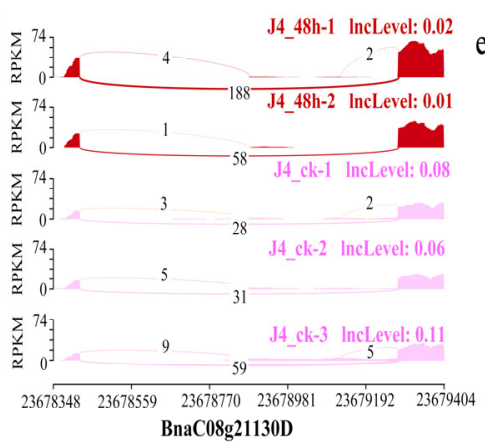

e)

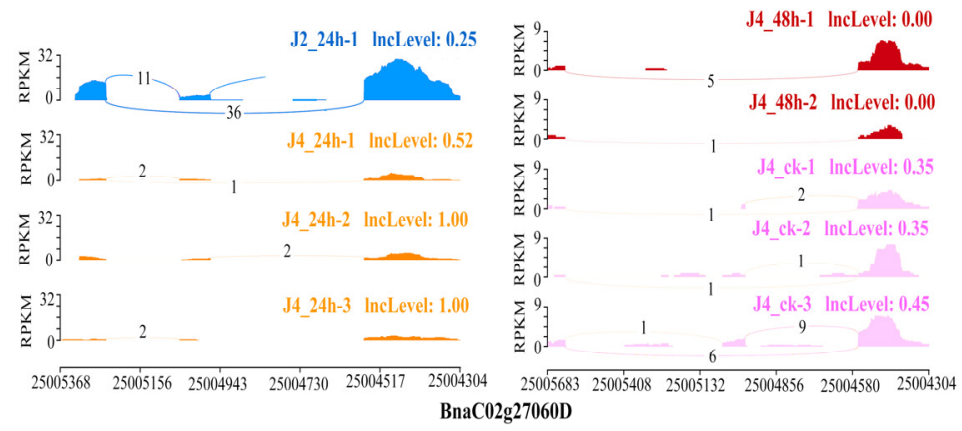

c)

f)

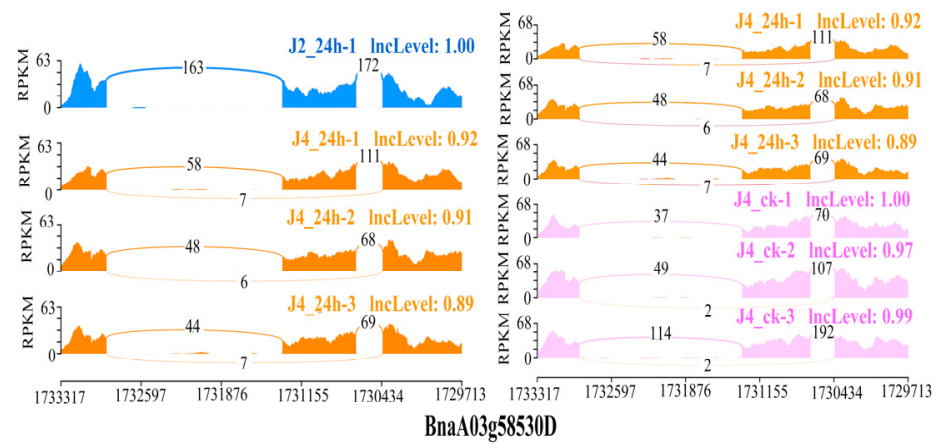

Figure 5. Quantitative visualization (sashimiplot) of the six candidate DAS genes. (a-c) illustrates the DAS between two groups of BnaC05g05550D, BnaC08g21130D and BnaC05g00740D. (d-f) illustrates the DAS between four groups of BnaA06g38980D, BnaC02g27060D and BnaA03g58530D. The different colors represent five different conditions: blue, cv. J902 at 24 hpi; red, cv. J964 at 48 hpi; pink, cv. J964 mock inoculation (ck); green, cv. J902 mock inoculation; and orange, cv. J964 at 24 hpi. The y axis represents a modified RPKM value. The $\mathrm{x}$ axis represents the genomic coordinates of each DAS gene. The numbers on the curved lines indicate the counts of each splice junction. The IncLevel value represents the normalized proportion of EI (Exon Inclusion) events. 


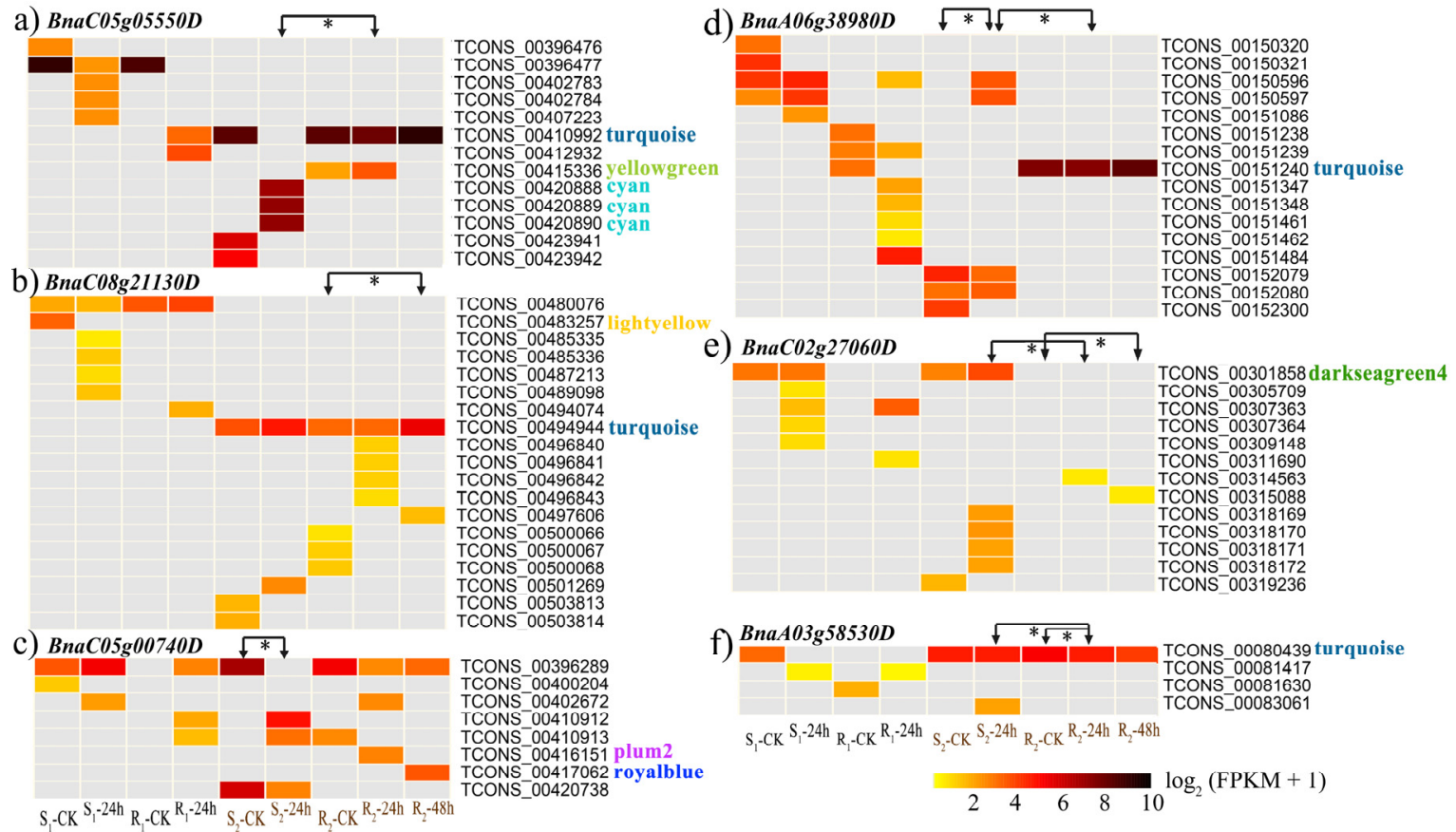

Figure 6. Expression heatmaps of each isoform of the six candidate DAS genes. (a-k) represents the isoform expression pattern of BnaC05g05550D, BnaC08g21130D, BnaC05g00740D, BnaA06g38980D, $B n a C 02 g 27060 D$ and BnaA03g58530D. The arrows indicate the materials in which the DAS genes are significantly differently abundant. The colors written behind the isoforms represent their involved coexpression modules. $\mathrm{S}_{1}-\mathrm{CK}$, mock-inoculated Westar plants; $\mathrm{S}_{1}-24 \mathrm{~h}$, Westar plants at $24 \mathrm{hpi}$; $\mathrm{R}_{1}-\mathrm{CK}$, mock-inoculated ZY821 plants; $\mathrm{R}_{1}-24 \mathrm{~h}, \mathrm{ZY} 821$ plants at 24 hpi; $\mathrm{S}_{2}-\mathrm{CK}$, mock-inoculated J902 plants; $\mathrm{S}_{2}-24 \mathrm{~h}$, J902 plants at $24 \mathrm{hpi} ; \mathrm{R}_{2}-\mathrm{CK}$, mock-inoculated J964 plants; $\mathrm{R}_{2}-24 \mathrm{~h}$, J964 plants at 24 hpi; $\mathrm{R}_{2-} 48 \mathrm{~h}$, J964 plants at $48 \mathrm{hpi}$.

BnaA06g38980D is homologous to AT3G44300, which encodes nitrilase 2 (NIT2). This gene was DAS in the susceptible and resistant B. napus cultivars at 24 hpi with S. sclerotiorum, and between the mock-treated and $24 \mathrm{hpi} \mathrm{susceptible} \mathrm{cultivar} \mathrm{plants} \mathrm{(Figure} \mathrm{5d).} \mathrm{In} \mathrm{the} \mathrm{infected} \mathrm{susceptible} \mathrm{cultivar} \mathrm{J2,}$ the IncLevel was much lower than in the infected resistant cultivar J4, suggesting that the resistant cultivar tended to reduce the number of ES events to retain as many exons as possible. The susceptible cultivar J2 had a much higher IncLevel when infected with S. sclerotiorum for $24 \mathrm{~h}$ than it did in the mock-treated condition, suggesting that it tends to reduce the ES events to retain all exons during infection. Expression heatmaps were made of all 16 isoforms of BnaA06g38980D (Figure 6d), enabling the identification of five isoforms that significantly differed in abundance between the different experimental conditions. After infection, TCONS_00150596 and TCONS_00150597 were detected in the susceptible cultivar, whereas TCONS_00151240 from the turquoise module only occurred in the resistant cultivar, suggesting that the isoforms that are specific to the cultivars may be key factors in the defense response.

BnaC02g27060D is homologous to AT4G02620, which encodes the vacuolar ATPase subunit F family protein (V-ATPase F). This gene was DAS in the mock-treated resistant cultivar and the same plants at 48 hpi with S. sclerotiorum, and between the two different cultivars at 24 hpi (Figure 5e). In the susceptible cultivar J2 at $24 \mathrm{hpi}$, the IncLevel was much higher than it was in the infected resistant cultivar J4, suggesting that the retention of the second exon in the resistant cultivar resulted in its high level of resistance to this pathogen. When compared with the mock-treated plants, the IncLevel of V-ATPase F was much lower in the resistant cultivar at $48 \mathrm{hpi}$, suggesting that infection may stimulate the ES events that generate the diverse isoforms. Expression heatmaps were made of all 13 isoforms of BnaC02g27060D (Figure 6e). Six isoforms were significantly differently abundant in the different materials. TCONS_00301858 was included in the darkseagreen4 module expressed in all 
the susceptible cultivars, but not in the resistant cultivars, which may suggest that this isoform was specific to the susceptible cultivar. None of the isoforms were expressed in the mock-treated resistant cultivars, indicating that V-ATPase F was only expressed in the resistant cultivars when induced by the pathogen.

BnaA03g58530D is homologous to AT4G21120, which encodes an amino acid transporter 1 (AAT1). This gene was DAS in the mock-treated and infected resistant cultivar J4 at 24 hpi, and between the susceptible and resistant cultivars at $24 \mathrm{hpi}$ (Figure 5f). Compared with the infected resistant cultivar, the infected susceptible cultivar and the mock-treated resistant cultivar both had higher IncLevels. More ES events may therefore occur in the infected resistant cultivar, which may therefore play a greater role in the infection response. Expression heatmaps were made of all four isoforms of BnaA03g58530D (Figure 6f). Two of these isoforms were significantly differently abundant in the different materials. TCONS_00080439 from the turquoise module occurred in both of the cultivar-related and infection-induced DAS groups, suggesting that it might be a key isoform responsible for enhancing resistance.

\section{Discussion and Conclusions}

In this study, we aimed to identify genetic factors and specific response mechanisms that influence resistance to SSR by analyzing the RNA-seq data of infected and mock-treated resistant and susceptible cultivars. Two types of analysis were performed: post-transcriptional differences were explored using a DAS analysis, and isoform-level transcriptional regulation was elucidated using a coexpression network analysis. A total of 79 DAS genes were identified, which was fewer than expected. However, a previous study showed that the use of ten differential splicing analysis tools resulted in the identification of different numbers of DAS genes. The number of identified DAS genes ranged from 0 (cuffdiff2) to 4506 (edgeR) in a human prostate cancer dataset, with 2962 and 0 DAS genes identified using rMATS in human and mouse datasets, respectively [49]. In addition, 252, 171, and 42 DAS genes were identified in comparisons between control and intolerant, control and tolerant and intolerant and tolerant catfish following heat stress [50]. Additionally, rMATS was used to identify 24, 40, and 74 DAS genes in the root, juvenile leaves and old leaves of B. napus, respectively, in response to boron deficiency [51].

The 79 DAS genes identified here were mainly involved in the nuclear retention of unspliced premRNA and the nuclear mRNA surveillance of the spliceosome. Using the same double-end RNA-seq data here, it has been reported that 13,276 differentially expressed genes (DEGs) participated in metabolic processes and responses to stimuli such as response to chitin, RNA methylation, secondary cell wall biogenesis and gravitropism [31]. However, these genes had little overlap with the 79 DAS genes identified here. A small overlap was identified between the DEGs and DAS genes in rice plants exposed to mineral (phosphorus, zinc, manganese, copper, and iron) deficiencies [52]. These comparisons suggested that AS is an additional layer of regulation, complementary to DEGs, that enables plants to respond to their environment.

S. sclerotiorum infects $B$. napus using mycelia, or through the production of apothecia which are laden with fungal spores [53]. The fungus produces multiple pathogenicity factors that attack B. napus, such as oxalic acid and an array of lytic enzymes [54]. When attacked by fungi, plants undergo a three-step process: pathogen perception, signal transduction and the defense response. In the plant-Sclerotinia system, pathogen detection is based on the recognition of pathogen-associated molecular patterns (PAMPs) by pattern recognition receptors, such as RLP30 (receptor-like protein 30) [7] and other receptor-like kinases, or via cytoplasmic leucine rich proteins (LRRs) [55]. The subsequent signal transduction involves a MAPK (mitogen-activated protein kinase) cascade or MAPK-independent pathways [56] involving many signaling molecules, including reactive oxygen species (ROS) [57], nitric oxide [58], salicylic acid [59], jasmonic acid and ethylene [60]. These signals drive plant PAMP-triggered immunity through the production of nuclear proteins including transcription factors and protein kinases that activate ROS production, detoxification, oxidative protection, callose deposition, camalexin production and the production of other specialized metabolites $[61,62]$. The DAS genes identified here, 
such as the immune-associated genes and those encoding important enzymes, might play a major role in the response to $S$. sclerotiorum.

Among the 79 DAS genes, we identified several genes, including SR, that are involved in RNA splicing. As constitutive and AS regulators, SR proteins consist of RNA recognition motif and a Ser/Arg-rich domain, which, respectively, recognize RNA and the spliceosome [63]. It has been reported that genes encoding SR proteins in plants appear to function mainly in stress responses; for example, in arabidopsis, the $s r 34 b$ T-DNA insertion mutant had a lower cadmium tolerance with a splicing defect in its iron-regulated transporter 1 [64], whereas the sr45 mutant was hypersensitive to glucose and abscisic acid [65]. In rice, the SR40, SCL57, and SCL25 proteins were important for phosphorus uptake and remobilization [52]. Recent studies have reported the stress-triggering AS pattern of certain $S R$ transcripts, which may contain premature termination codons, resulting in their degradation by the nonsense-mediated decay pathway. For example, the abundance of an unproductive premature termination codon-containing AS isoform of arabidopsis SR30 decreases at elevated temperatures [66]. The AS of SR genes can increase plant tolerance to hormones and stress treatments in rice and $B$. napus $[51,67,68]$. It was interesting to discover that the gene encoding the splicing factor PRP18 protein (BnaA09g50970D) is an overlapping DAS gene, as the homologous mutant in $A$. thaliana produces short roots and small siliques following its induction of large genome-wide IR events [45]. Additionally, BnaCnn1978, encoding an RNA binding protein, was identified as an infection-induced DAS gene. The splicing-related gene SR34a was also involved in two defense-related modules, and the increased number of ES events at 48 hpi with S. sclerotiorum suggests that this crucial splicing factor might be activated in response to pathogen stress to regulate downstream networks. The regulation relationships should be verified in further experiments.

In addition to SR34a, we also identified other five DAS genes (APX1, NIT2, AAT1, V-ATPase F and a gene encoding an unknown protein) as crucial candidate genes in the response to pathogen defense. APX isoenzymes function in controlling ROS metabolism and eliminating $\mathrm{H}_{2} \mathrm{O}_{2}$ [69]. APX can respond to environmental stresses such as oxidative stresses [70,71], light stress [69] and pathogen infection (Trichoderma harzianum) [72,73]. In this study, we identified APX1 as a cultivar-related DAS with isoforms involved in different coexpression networks in the response to pathogen stress. NIT2 in $A$. thaliana is able to hydrolyze a broad substrate range [74], whereas a recent study revealed that AtNIT2 expression was de novo-induced by a plant pathogen (Pseudomonas syringae pv. tomato) and responsible for R gene-mediated resistance responses [75]. We established that BnNIT2 was differently spliced in the susceptible cultivar at 24 hpi compared with the mock-treated plants, and seemed to reduce the ES events in the susceptible cultivar in response to $S$. sclerotiorum. V-ATPase F proton pumps are responsible for the ATP hydrolysis that drives ion transport [76]. It has been reported that V-ATPase functions in response to phosphorus deficiency [77], copper stress [76], fungal infection (the dermatophyte Trichophyton rubrum) [78] among other stresses. We identified V-ATPase F as a pathogen-induced and cultivar-related DAS gene, and its AS frequency increased at $48 \mathrm{hpi}$. AATs are responsible for the long-distance transport of amino acids from the root to the shoot [79]. The overexpression of AtAAT1 resulted in improved resistance against the pathogen Pseudomonas syringae [80]. In this study, we identified AAT1 as both a pathogen-induced and cultivar-related DAS gene, which might lead to the generation of more AS events at $24 \mathrm{hpi}$ with S. sclerotiorum to increase plant resistance to this pathogen.

In this study, a total of 79 DAS genes were identified in B. napus after inoculation with S. sclerotiorum, and a combination of a DAS analysis and an isoform WGCNA revealed six candidate genes with important putative functions in pathogen resistance. These observations enhance our understanding of the post-transcriptional response of $B$. napus to $S$. sclerotiorum infection and pave the way to developing rapeseed lines with improved resistance to this devastating pathogen. Continuous advances in molecular biological experiments in exploring the function of different isoforms of one single gene should facilitate our findings of the candidate isoforms in pathogen defense.

Supplementary Materials: The following are available online at http://www.mdpi.com/2073-4425/11/7/784/s1, Table S1: FPKM values of 575172 isoforms in 18 samples. Table S2: The mapped reads to reference genome and 
mapped read length of 18 RNA-seq raw data. Table S3: The AS patterns of various plant materials. Table S4: Differential alternative splicing analyses using rMATs. Table S5: Enriched GO terms of 79 DAS genes. Table S6: Network nodes of every module gained from WGCNA. Table S7: Enriched GO terms of genes in the 85 WGCNA modules. Table S8: The node relationships of 11 co-expression networks by Cytoscape. Figure S1: Enriched GO terms of 79 DAS genes. Figure S2 Enriched GO terms of genes in the 85 WGCNA modules.

Author Contributions: Conceptualization, J.-Q.M. and L.-J.W.; Methodology, J.Q.M. and W.X.; Formal analysis, W.X. and W.S.; Software H.-H.J. and W.S.; Writing-original draft preparation, J.-Q.M and W.X.; Writing-review and editing, L.-J.W.; Visualization, F.X. and A.L.; Supervision, K.L., L.-J.W. and J.N.L.; Funding acquisition, L.-J.W. and J.-N.L. All authors have read and agreed to the published version of the manuscript.

Funding: This research was funded by the National Natural Science Foundation of China (31701460) and the "111" Project (B12006).

Conflicts of Interest: The authors declare no conflict of interest.

\section{References}

1. Chalhoub, B.; Denoeud, F.; Liu, S.; Parkin, I.A.; Tang, H.; Wang, X.; Chiquet, J.; Belcram, H.; Tong, C.; Samans, B.; et al. Early allopolyploid evolution in the post-Neolithic Brassica napus oilseed genome. Science (New York) 2014, 345, 950-953. [CrossRef] [PubMed]

2. Liu, L.; Free, S.J. Characterization of the Sclerotinia sclerotiorum cell wall proteome. Mol. Plant Pathol. 2015, 17, 985-995. [CrossRef] [PubMed]

3. Farr, D.F.; Rossman, A.Y. Fungal Databases, U.S. National Fungus Collections, ARS, USDA. Available online: https://nt.ars-grin.gov/fungaldatabases/ (accessed on 9 July 2020).

4. Bolton, M.D.; Thomma, B.P.H.J.; Nelson, B.D. Sclerotinia sclerotiorum (Lib.) de Bary: Biology and molecular traits of a cosmopolitan pathogen. Mol. Plant Pathol. 2006, 7, 1-16. [CrossRef] [PubMed]

5. Derbyshire, M.; Denton-Giles, M. The control of Sclerotinia stem rot on oilseed rape ( Brassica napus ): Current practices and future opportunities. Plant Pathol. 2016, 2016. 65, 859-877. [CrossRef]

6. Wang, Z.; Ma, L.Y.; Cao, J.; Li, Y.L.; Ding, L.N.; Zhu, K.M.; Yang, Y.H.; Tan, X.L. Recent Advances in Mechanisms of Plant Defense to Sclerotinia sclerotiorum. Front. Plant Sci. 2019, 10, 1314. [CrossRef] [PubMed]

7. Jones, J.D.G.; Dangl, J.L. The plant immune system. Nature 2006, 2006. 444, 323-329. [CrossRef]

8. Fenton, A.; Antonovics, J.; Brockhurst, M.A. Inverse-Gene-for-Gene Infection Genetics and Coevolutionary Dynamics. Am. Nat. 2009, 2009. 174, E230-E242. [CrossRef]

9. Derbyshire, M.; Mbengue, M.; Barascud, M.; Navaud, O.; Raffaele, S. Small RNAs from the plant pathogenic fungus Sclerotinia sclerotiorum highlight host candidate genes associated with quantitative disease resistance. Mol. Plant Pathol. 2019, 20, 1279-1297. [CrossRef] [PubMed]

10. Wu, J.; Zhao, Q.; Liu, S.; Shahid, M.; Lan, L.; Cai, G.; Zhang, C.; Fan, C.; Wang, Y.; Zhou, Y. Genome-wide Association Study Identifies New Loci for Resistance to Sclerotinia Stem Rot in Brassica napus. Front. Plant Sci. 2016, 7, 1418. [CrossRef] [PubMed]

11. Wei, D.; Mei, J.; Fu, Y.; Disi, J.O.; Li, J.; Qian, W. Quantitative trait loci analyses for resistance to Sclerotinia sclerotiorum and flowering time in Brassica napus. Mol. Breed. 2014, 34, 1797-1804. [CrossRef]

12. Girard, I.J.; Tong, C.; Becker, M.G.; Mao, X.; Huang, J.; de Kievit, T.; Fernando, W.D.; Liu, S.; Belmonte, M.F. RNA sequencing of Brassica napus reveals cellular redox control of Sclerotinia infection. J. ExBot. 2017. [CrossRef] [PubMed]

13. Joshi, R.K.; Megha, S.; Basu, U.; Rahman, M.H.; Kav, N.N.V. Genome Wide Identification and Functional Prediction of Long Non-Coding RNAs Responsive to Sclerotinia sclerotiorum Infection in Brassica napus. PLoS ONE 2016, 11, e0158784. [CrossRef] [PubMed]

14. Calixto, C.P.G.; Guo, W.; James, A.B.; Tzioutziou, N.A.; Brown, J.W.S. Rapid and Dynamic Alternative Splicing Impacts the Arabidopsis Cold Response Transcriptome. Plant Cell 2018, 30, 1424-1444. [CrossRef] [PubMed]

15. Wang, Y.; Liu, J.; Huang, B.O.; Xu, Y.M.; Li, J.; Huang, L.F.; Lin, J.; Zhang, J.; Min, Q.H.; Yang, W.M.; et al. Mechanism of alternative splicing and its regulation. Biomed. Rep. 2015, 3, 152-158. [CrossRef] [PubMed]

16. Syed, N.H.; Kalyna, M.; Marquez, Y.; Barta, A.; Brown, J.W.S. Alternative splicing in plants-coming of age. Trends Plant Sci. 2012, 17, 616-623. [CrossRef] [PubMed] 
17. Liu, Z.; Qin, J.; Tian, X.; Xu, S.; Wang, Y.; Li, H.; Wang, X.; Peng, H.; Yao, Y.; Hu, Z.; et al. Global profiling of alternative splicing landscape responsive to drought, heat and their combination in wheat (Triticum aestivum L.). Plant Biotechnol. J. 2018, 16, 714-726. [CrossRef]

18. Ma, J.Q.; Wei, L.J.; Lin, A.; Zhang, C.; Sun, W.; Yang, B.; Lu, K.; Li, J.N. The Alternative Splicing Landscape of Brassica napus Infected with Leptosphaeria maculans. Genes 2019, 10, 296. [CrossRef]

19. Mandadi, K.K.; Scholthof, K.B.G. Genome-Wide Analysis of Alternative Splicing Landscapes Modulated during Plant-Virus Interactions in Brachypodium distachyon. Plant Cell 2015, 27, 71-85. [CrossRef]

20. Gil, A. How did alternative splicing evolve? Nat. Rev. Genet. 2004, 5, 773-782. [CrossRef]

21. Baralle, F.E.; Giudice, J. Alternative splicing as a regulator of development and tissue identity. Nat. Rev. Mol. Cell Biol. 2017, 18, 437. [CrossRef]

22. Breathnach, R.; Benoist, C.; O'Hare, K.; Gannon, F.; Chambon, P. Ovalbumin Gene: Evidence for a Leader Sequence in mRNA and DNA Sequences at the Exon-Intron Boundaries. Proc. Natl. Acad. Sci. 1978, 75, 4853-4857. [CrossRef] [PubMed]

23. Staiger, D.; Brown, J.W.S. Alternative splicing at the intersection of biological timing, development, and stress responses. Plant Cell 2013, 25, 3640-3656. [CrossRef] [PubMed]

24. Fu, X.D.; Ares, M. Context-dependent control of alternative splicing by RNA-binding proteins. Nat. Rev. Genet. 2014, 15, 689-701. [CrossRef] [PubMed]

25. Li, W.; Lin, W.-D.; Ray, P.; Lan, P.; Schmidt, W. Genome-Wide Detection of Condition-Sensitive Alternative Splicing in Arabidopsis Roots. Plant Physiol. 2013, 162, 1750-1763. [CrossRef] [PubMed]

26. Krawczak, M.; Reiss, J.; Cooper, D.N. The mutational spectrum of single base-pair substitutions in mRNA splice junctions of human genes: Causes and consequences. Hum. Genet. 1992, 90, 41-54. [CrossRef]

27. Yang, S.; Tang, F.; Zhu, H. Alternative Splicing in Plant Immunity. Int. J. Mol. Sci. 2014, 15, 10424-10445. [CrossRef]

28. Dinesh-Kumar, S.P.; Baker, B.J. Alternatively spliced N resistance gene transcripts: Their possible role in tobacco mosaic virus resistance. Proc. Natl. Acad. Sci. USA 2000, 97, 1908-1913. [CrossRef]

29. Zhang, X.-C.; Gassmann, W. Alternative splicing and mRNA levels of the disease resistance gene RPS4 are induced during defense responses. Plant Physiol. 2007, 145, 1577-1587. [CrossRef]

30. Liu, J.; Chen, X.; Liang, X.; Zhou, X.; Yang, F.; Liu, J.; He, S.Y.; Guo, Z. Alternative splicing of rice WRKY62 and WRKY76 transcription factor genes in pathogen defense. Plant Physiol. 2016, 171, 1427-1442. [CrossRef]

31. Wu, J.; Zhao, Q.; Yang, Q.; Liu, H.; Li, Q.; Yi, X.; Cheng, Y.; Guo, L.; Fan, C.; Zhou, Y. Comparative transcriptomic analysis uncovers the complex genetic network for resistance to Sclerotinia sclerotiorum in Brassica napus. Sci. Rep. 2016, 6, 19007. [CrossRef]

32. Bolger, A.M.; Lohse, M.; Usadel, B. Trimmomatic: A flexible trimmer for Illumina sequence data. Bioinformatics 2014, 30, 2114-2120. [CrossRef] [PubMed]

33. Dobin, A.; Davis, C.A.; Schlesinger, F.; Drenkow, J.; Zaleski, C.; Jha, S.; Batut, P.; Chaisson, M.; Gingeras, T.R. STAR: Ultrafast universal RNA-seq aligner. Bioinformatics 2013, 29, 15-21. [CrossRef] [PubMed]

34. Trapnell, C.; Roberts, A.; Goff, L.; Pertea, G.; Kim, D.; Kelley, D.R.; Pimentel, H.; Salzberg, S.L.; Rinn, J.L.; Pachter, L. Differential gene and transcript expression analysis of RNA-seq experiments with TopHat and Cufflinks. Nat. Protoc. 2012, 7, 562-578. [CrossRef] [PubMed]

35. Sylvain, F.; Michael, S. ASTALAVISTA: Dynamic and flexible analysis of alternative splicing events in custom gene datasets. Nucleic Acids Res. 2007, 35 (Suppl. 2), W297-W299. [CrossRef]

36. Shen, S.; Park, J.W.; Lu, Z.X.; Lin, L.; Henry, M.D.; Wu, Y.N.; Zhou, Q.; Xing, Y. rMATS: Robust and flexible detection of differential alternative splicing from replicate RNA-Seq data. Proc. Natl. Acad. Sci. 2014, 111, E5593-E5601. [CrossRef] [PubMed]

37. Langfelder, P.; Horvath, S. WGCNA: An R package for weighted correlation network analysis. BMC Bioinformatics 2008, 9, 559. [CrossRef]

38. Zhang, B.; Horvath, S. A General Framework For Weighted Gene Co-Expression Network Analysis. Stat. Appl. Genet. Mol. Biol. 2005, 4, 17. [CrossRef]

39. Kohl, M.; Wiese, S.; Warscheid, B. Cytoscape: Software for Visualization and Analysis of Biological Networks. Methods Mol. Biol. 2011, 696, 291-303. [CrossRef]

40. Alexa, A.; Rahnenführer, J.; Lengauer, T. Improved scoring of functional groups from gene expression data by decorrelating go graph structure. Bioinformatics 2006, 22, 1600-1607. [CrossRef] 
41. Schranz, M.E.; Lysak, M.A.; Mitchell-Olds, T. The ABC's of comparative genomics in the Brassicaceae: Building blocks of crucifer genomes. Trends Plant Sci. 2006, 11, 535-542. [CrossRef]

42. Murat, F.; Louis, A.; Maumus, F.; Armero, A.; Cooke, R.; Quesneville, H.; Crollius, H.R.; Salse, J. Understanding Brassicaceae evolution through ancestral genome reconstruction. Genome Biol. 2015, 16, 262. [CrossRef] [PubMed]

43. Camacho, C.; Coulouris, G.; Avagyan, V.; Ma, N.; Papadopoulos, J.; Bealer, K.; Madden, T.L. BLAST+: Architecture and applications. BMC Bioinform. 2009, 10, 421. [CrossRef] [PubMed]

44. Wang, B.-B.; Brendel, V. Genomewide comparative analysis of alternative splicing in plants. Proc. Natl. Acad. Sci. USA 2006, 103, 7175-7180. [CrossRef] [PubMed]

45. Kanno, T.; Lin, W.-D.; Chang, C.-L.; Matzke, M.; Matzke, A.J.M. A Genetic Screen Identifies PRP18a, a Putative Second Step Splicing Factor Important for Alternative Splicing and a Normal Phenotype in Arabidopsis thaliana. G3: Genes Genomes Genet. 2018, 8, 1367-1377. [CrossRef] [PubMed]

46. Wang, Y.; Li, Y.; Rosas-Diaz, T.; Caceres-Moreno, C.; Lozano-Duran, R.; Macho, A.P. The IMMUNE-ASSOCIATED NUCLEOTIDE-BINDING 9 Protein Is a Regulator of Basal Immunity in Arabidopsis thaliana. Mol. Plant. Microbe Interact. 2019, 32, 65-75. [CrossRef] [PubMed]

47. Wu, Z.; Li, M.; Dong, O.X.; Xia, S.; Liang, W.; Bao, Y.; Wasteneys, G.; Li, X. Differential regulation of TNL-mediated immune signaling by redundant helper CNLs. New Phytol. 2019, 222, 938-953. [CrossRef]

48. Majewska, M.; Lipka, A.; Paukszto, L.; Jastrzebski, J.P.; Szeszko, K.; Gowkielewicz, M.; Lepiarczyk, E.; Jozwik, M.; Majewski, M.K. Placenta Transcriptome Profiling in Intrauterine Growth Restriction (IUGR). Int. J. Mol. Sci. 2019, 20, 1510. [CrossRef]

49. Mehmood, A.; Laiho, A.; Venäläinen, M.S.; McGlinchey, A.J.; Wang, N.; Elo, L.L. Systematic evaluation of differential splicing tools for RNA-seq studies. Brief. Bioinform. 2019. [CrossRef]

50. Tan, S.; Wang, W.; Tian, C.; Niu, D.; Zhou, T.; Jin, Y.; Yang, Y.; Gao, D.; Dunham, R.; Liu, Z. Heat stress induced alternative splicing in catfish as determined by transcriptome analysis. Comp. Biochem. Physiol. Part D: Genom. Proteom. 2019, 29, 166-172. [CrossRef]

51. Gu, J.; Li, W.; Wang, S.; Zhang, X.; Coules, A.; Ding, G.; Xu, F.; Ren, J.; Lu, C.; Shi, L. Differential Alternative Splicing Genes in Response to Boron Deficiency in Brassica napus. Genes 2019, 10, 224. [CrossRef]

52. Dong, C.; He, F.; Berkowitz, O.; Liu, J.; Cao, P.; Tang, M.; Shi, H.; Wang, W.; Li, Q.; Shen, Z. Alternative Splicing Plays a Critical Role in Maintaining Mineral Nutrient Homeostasis in Rice (Oryza sativa). Plant Cell 2018, 30, 2267-2285. [CrossRef] [PubMed]

53. Clarkson, J.P.; Phelps, K.; Whipps, J.M.; Young, C.S.; Smith, J.A.; Watling, M. Forecasting Sclerotinia Disease on Lettuce: A Predictive Model for Carpogenic Germination of Sclerotinia sclerotiorum Sclerotia. Phytopatholog 2007, 97, 621-631. [CrossRef] [PubMed]

54. Sharma, P.; Meena, P.D.; Verma, P.R.; Saharan, G.S.; Mehta, N.; Singh, D.; Kumar, A. Sclerotinia sclerotiorum (Lib.) de Bary causing Sclerotinia rot in oilseed Brassicas: A review. J. Oilseed Brassica 2016, 6, 1-44.

55. Corwin, J.A.; Kliebenstein, D.J. Quantitative Resistance: More than just perception of a pathogen. Plant Cell 2017, 29, 655-665. [CrossRef] [PubMed]

56. Wei, M.; Berkowitz, G.A. Ca2+ conduction by plant cyclic nucleotide gated channels and associated signaling components in pathogen defense signal transduction cascades. New Phytol. 2011, 190. [CrossRef]

57. Arfaoui, A.; Hadrami, A.E.; Daayf, F. Pre-treatment of soybean plants with calcium stimulates ROS responses and mitigates infection by Sclerotinia sclerotiorum. Plant Physiol. Biochem. 2017, 122, 121-128. [CrossRef]

58. Arasimowicz-Jelonek, M.; Floryszak-Wieczorek, J. Nitric Oxide in the Offensive Strategy of Fungal and Oomycete Plant Pathogens. Front. Plant Sci. 2016, 7, 252. [CrossRef]

59. Nováková, M.; Šašek, V.; Dobrev, P.I.; Valentová, O.; Burketová, L. Plant hormones in defense response of Brassica napus to Sclerotinia sclerotiorum-Reassessing the role of salicylic acid in the interaction with a necrotroph. Plant Physiol. Biochem. 2014, 80, 308-317. [CrossRef]

60. Perchepied, L.; Balagué, C.; Riou, C.; Claudel-Renard, C.; Rivière, N.; Grezes-Besset, B.; Roby, D. Nitric oxide participates in the complex interplay of defense-related signaling pathways controlling disease resistance to Sclerotinia sclerotiorum in Arabidopsis thaliana. Mol. Plant. Microbe Interact. 2010, 23, 846. [CrossRef]

61. Xu, Y.-P.; Yang, J.; Cai, X.-Z. Glycolate oxidase gene family in Nicotiana benthamiana: Genome-wide identification and functional analyses in disease resistance. Sci. Rep. 2018, 8, 8615. [CrossRef] 
62. Corwin, J.A.; Copeland, D.; Feusier, J.; Subedy, A.; Eshbaugh, R.; Palmer, C.; Maloof, J.; Kliebenstein, D.J. The Quantitative Basis of the Arabidopsis Innate Immune System to Endemic Pathogens Depends on Pathogen Genetics. PLoS Genet. 2016, 12, e1005789. [CrossRef] [PubMed]

63. Joris, M.; Schloesser, M.; Baurain, D.; Hanikenne, M.; Muller, M.; Motte, P. Number of inadvertent RNA targets for morpholino knockdown in Danio rerio is largely underestimated: Evidence from the study of Ser/Arg-rich splicing factors. Nucleic Acids Res. 2017, 45, 9547-9557. [CrossRef] [PubMed]

64. Zhang, W.; Du, B.; Liu, D.; Qi, X. Splicing factor SR34b mutation reduces cadmium tolerance in Arabidopsis by regulating iron-regulated transporter 1 gene. Biochem. Biophys. Res. Commun. 2014, 455, 312-317. [CrossRef] [PubMed]

65. Carvalho, R.F.; Carvalho, S.D.; Duque, P. The plant-specific SR45 protein negatively regulates glucose and ABA signaling during early seedling development in Arabidopsis. Plant Physiol. 2010, 154, 772-783. [CrossRef]

66. Filichkin, S.A.; Priest, H.D.; Givan, S.A.; Shen, R.; Bryant, D.W.; Fox, S.E.; Wong, W.K.; Mockler, T.C. Genome-wide mapping of alternative splicing in Arabidopsis thaliana. Genome Res. 2010, 20, 45-58. [CrossRef] [PubMed]

67. Isshiki, M.; Tsumoto, A.; Shimamoto, K. The serine/arginine-rich protein family in rice plays important roles in constitutive and alternative splicing of pre-mRNA. Plant Cell 2006, 18, 146-158. [CrossRef] [PubMed]

68. Peng, Z.; Heng, D.; Fang-ming, X.; Yong-sheng, L. Alterations of Alternative Splicing Patterns of Ser/Arg-Rich (SR) Genes in Response to Hormones and Stresses Treatments in Different Ecotypes of Rice (Oryza sativa). J. Integr. Agric. 2013, 12, 737-748. [CrossRef]

69. Davletova, S.; Rizhsky, L.; Liang, H.; Shengqiang, Z.; Oliver, D.J.; Coutu, J.; Shulaev, V.; Schlauch, K.; Mittler, R. Cytosolic ascorbate peroxidase 1 is a central component of the reactive oxygen gene network of Arabidopsis. Plant Cell 2005, 17, 268-281. [CrossRef]

70. Caverzan, A.; Passaia, G.; Rosa, S.B.; Ribeiro, C.W.; Lazzarotto, F.; Margis-Pinheiro, M. Plant responses to stresses: Role of ascorbate peroxidase in the antioxidant protection. Genet. Mol. Biol. 2012, 35 (Suppl. 4), 1011-1019. [CrossRef]

71. Akram, N.A.; Shafiq, F.; Ashraf, M. Ascorbic Acid-A Potential Oxidant Scavenger and Its Role in Plant Development and Abiotic Stress Tolerance. Front. Plant Sci. 2017, 8, 613. [CrossRef]

72. Mittler, R.; Feng, X.; Cohen, M. Post-transcriptional suppression of cytosolic ascorbate peroxidase expression during pathogen-induced programmed cell death in tobacco. Plant Cell 1998, 10, 461-473. [CrossRef] [PubMed]

73. Zehra, A.; Meena, M.; Dubey, M.K.; Aamir, M.; Upadhyay, R.S. Synergistic effects of plant defense elicitors and Trichoderma harzianum on enhanced induction of antioxidant defense system in tomato against Fusarium wilt disease. Bot. Stud. 2017, 58, 44. [CrossRef] [PubMed]

74. Schreiner, U.; Steinkellner, G.; Rozzell, J.D.; Glieder, A.; Winkler, M. Improved Fitness of Arabidopsis thaliana Nitrilase 2. Chem CatChem 2010, 2, 263-267. [CrossRef]

75. Choi du, S.; Lim, C.W.; Hwang, B.K. Proteomics and functional analyses of Arabidopsis nitrilases involved in the defense response to microbial pathogens. Planta 2016, 244, 449-465. [CrossRef]

76. Morris, C.A.; Owen, J.R.; Thomas, M.C.; EL-HITI, G.A.; Harwood, J.L.; Kille, P. Intracellular localization and induction of a dynamic RNA-editing event of macro-algal V-ATPase subunit A (VHA-A) in response to copper. Plant Cell Environ. 2014, 37, 189-203. [CrossRef]

77. Lin, Z.H.; Chen, L.S.; Chen, R.B.; Zhang, F.Z.; Yang, L.T.; Tang, N. Expression of genes for two phosphofructokinases, tonoplast ATPase subunit A, and pyrophosphatase of tea roots in response to phosphorus-deficiency. J. Horticult. Sci. Biotechnol. 2010, 85, 449-453. [CrossRef]

78. Rezaie, S.; Khodadadi, H.; Noorbakhsh, F.; Safari, M. Molecular Characterization of Subunit G of the Vacuolar ATPase in Pathogen Dermatophyte Trichophyton rubrum. Iran J. Public Health 2006, 35, 33-37.

79. Tegeder, M.; Rentsch, D. Uptake and partitioning of amino acids and peptides. Mol Plant 2010, 3, 997-1011. [CrossRef]

80. Yang, H.; Postel, S.; Kemmerling, B.; Ludewig, U. Altered growth and improved resistance of Arabidopsis against Pseudomonas syringae by overexpression of the basic amino acid transporter AtCAT1. Plant Cell Environ. 2014, 37, 1404-1414. [CrossRef]

(C) 2020 by the authors. Licensee MDPI, Basel, Switzerland. This article is an open access article distributed under the terms and conditions of the Creative Commons Attribution (CC BY) license (http://creativecommons.org/licenses/by/4.0/). 